\title{
VIGILÂNCIA EPIDEMIOLÓGICA E CONTROLE VETORIAL: IMPORTÂNCIA E DESAFIOS NA TRANSMISSÃO DA DOENÇA DE CHAGAS EM DUAS DÉCADAS NA REGIÃO DA ESTRADA DE FERRO DE GOIÁS
}

Maria Amélia Cândida Machado ${ }^{1}$, Matheus Brasileiro Alves ${ }^{2}$, Lanussy Porfiro de Oliveira $^{3}$, Thiago Sardinha de Oliveira ${ }^{4}$ e Heliana Batista de Oliveira ${ }^{5}$

${ }^{1}$ Mestranda em Educação pelo Programa de Pós-graduação em Educação da Universidade Federal de Catalão, Catalão-GO, Brasil.

${ }^{2}$ Graduando em Ciências Biológicas pela Universidade Federal de Catalão, CatalãoGO, Brasil.

${ }^{3}$ Doutoranda em Ciências Farmacêuticas pela Universidade Federal de Goiás, Goiânia-GO, Brasil.

${ }^{4}$ Professor do Curso de Farmácia da Universidade Federal dos Vales do Jequitinhonha e Mucuri, Diamantina-MG, Brasil. E-mail: thiago_tso@hotmail.com ${ }^{5}$ Professora do Curso de Ciências Biológicas da Universidade Federal de Catalão, Catalão-GO, Brasil.

Recebido em: 15/02/2020 - Aprovado em: 15/03/2020 - Publicado em: 30/03/2020 DOI: 10.18677/EnciBio_2020A24

A Doença de Chagas é uma parasitose causada pelo Trypanosoma cruzi e constitui um grave problema de saúde em regiões tropicais e subtropicais, apresentando as fases aguda e crônica. A transmissão vetorial é a mais conhecida, na qual as fezes infectadas pelo Trypanosoma cruzi penetram no orifício da picada do barbeiro, inseto vetor da doença. Com a descentralização das ações de controle de endemias e municipalização dos programas de controle de endemias, o presente trabalho objetivou levantar dados referentes à coleta de triatomíneos em municípios da Região de Saúde Estrada de Ferro - Macrorregião Centro Sudeste de Goiás nas últimas duas décadas, bem como delimitar a importância e os desafios encontrados em tal controle. Com o presente trabalho pode ser mostrado que a doença de chagas ainda representa um grande desafio público no Brasil e um problema de saúde emergente. Ações de educação em saúde juntamente com a vigilância entomológica e centralização na promoção da qualidade de vida da população são necessárias, a fim de evitar o processo de domiciliação de triatomíneos característicos exclusivamente de ambientes silvestres.

PALAVRAS-CHAVE: Doença de chagas, Trypanosoma cruzi, Vigilância epidemiológica. 


\title{
EPIDEMIOLOGICAL SURVEILLANCE AND VECTOR CONTROL: IMPORTANCE AND CHALLENGES IN TRANSMISSION OF CHAGAS DISEASE IN TWO DECADES IN REGION OF IRON ROAD OF GOIÁS
}

\begin{abstract}
Chagas disease is a parasitosis caused by Trypanosoma cruzi and constitutes a serious health problem in tropical and subtropical regions, presenting acute and chronic phases. Vector transmission is best known, which feces infected by Trypanosoma cruzi penetrate the orifice of barber's bite, insect vector of disease. With decentralization of endemic control actions and municipalization of endemic control programs, the present study aimed collect data regarding collection of triatomines in municipalities in iron road of Goiás in last two decades, as well as delimiting importance and challenges encountered in such control. This work shown that Chagas disease still represents a major public challenge in Brazil and emerging health problem. Health education actions together with entomological surveillance and centralization in promotion of population's quality of life are necessary in order to avoid process of domiciliation of triatomines characteristic exclusively of wild environments.
\end{abstract}

KEYWORDS: Chagas disease, Trypanosoma cruzi, Epidemiological surveillance.

\section{INTRODUÇÃO}

A Doença de Chagas (DC), parasitose causada pelo protozoário flagelado denominado Trypanosoma cruzi, constitui um grave problema de saúde em regiões tropicais e subtropicais (WHO, 2018). A infecção chagásica apresenta dois estágios para sua identificação, denominados fase aguda, no início da infecção, e a fase crônica levando algum tempo para ser diagnosticada. A fase aguda geralmente é assintomática, poucos pacientes apresentam sintomas em uma ou duas semanas após a infecção, desaparecendo rapidamente. Após algum tempo, inicia-se a fase crônica na forma indeterminada, não apresentando nenhuma manifestação clínica. Alguns pacientes permanecem por muitos anos, podendo levar até 20 anos para iniciar o aparecimento dos sintomas digestórios (megaesôfago e megacólon), cardíacos ou neurológicos (GUARNER., 2019). A fase crônica se caracteriza por ser assintomática ou com sintomatologia inespecífica, dificultando assim o diagnóstico que, na maioria das vezes, é realizado ocasionalmente por inquéritos sistemáticos (KRATZ, 2019).

A principal e mais conhecida forma de transmissão é a vetorial, no entanto outras formas de contaminação são comumente descritas como a via oral, a materno-fetal, sangue e hemoderivados, transplantes de órgão ou acidentes com material biológico (BRASIL, 2017). Outras formas de transmissão como oral, transplacentária, contaminação com caças silvestres e a presença de outras espécies de triatomíneos com potencial vetorial, contribuem para a continuidade do ciclo parasitário da tripanossomíase (GASPARIM et al., 2018).

Os insetos vetores, conhecidos popularmente por "barbeiros" ou "chupões" no Brasil, são hematófagos da subfamília Triatominae (Hemiptera: Reduviidae) (ARAÚJO et al., 2015). A transmissão vetorial ocorre no momento do repasto sanguíneo do triatomíneo para um hospedeiro ou reservatório. Em decorrência do aparelho digestório curto, o inseto defeca logo após a ingestão de sangue do hospedeiro vertebrado. As fezes contaminadas pelas formas evolutivas infectantes 
de $T$. cruzi (tripomastigotas metacíclicas) penetram no orifício da picada ou ativamente pelas mucosas (ARRUDA et al., 2019).

O controle da DC está baseado na captura, identificação e exames de vetores e na detecção de anticorpos IgG anti-Trypanosoma cruzi em bancos de doadores de sangue. Esses controles são realizados com sucesso no país e contribuíram para a diminuição de novos casos da doença de Chagas (DIAS, et al., 2015). O Brasil conseguiu, em 2006, validar a interrupção da transmissão vetorial do $T$. cruzi pelo Triatoma infestans, já foi considerado o principal vetor da DC, sendo a Bahia o último estado endêmico da doença a receber o certificado, enquanto Rio Grande do Sul, Tocantins, Paraná, São Paulo, Mato Grosso, Mato Grosso do Sul, Pernambuco, Minas Gerais, Piauí, Paraíba, Distrito Federal, Rio de Janeiro já haviam recebido tal certificação em momentos anteriores (SILVA, 2006). No entanto algumas espécies de triatomíneos estão apresentando potencial vetorial para DC, demonstrando a importância da continuidade do controle vetorial da doença (GASPARIM et al., 2018).

O controle da DC, com alcance nacional, foi assumido a partir dos avanços no controle da malária, e os excedentes de recursos foram realocados para o controle da endemia chagásica (DIAS et al., 2016). Desde 1999 a descentralização das ações de controle de endemias através do Programa de Controle de Doença de Chagas (PCDCh), assim as transferências das responsabilidades foram transferidas para os Estados e municípios através da Portaria ministerial №. 1.399 de 15 de dezembro de 1999 (BRASIL, 1999).

Com a municipalização, as prefeituras começaram a conduzir os programas de controle de endemias em Goiás sob a coordenação das Gerências Regionais de Saúde (GRS), sediadas em municípios de maior importância política ou econômica (GOIÁS, 2018). Diante da importância do controle vetorial para a interrupção da transmissão da DC e das modificações resolutivas sobre a responsabilidade do mesmo, o presente trabalho objetivou levantar os dados referentes à coleta de triatomíneos na Região de Saúde Estrada de Ferro - Macrorregião Centro Sudeste de Goiás nas últimas duas décadas, bem como delimitar a importância e os desafios encontrados em tal controle.

\section{MATERIAL E METODOS}

O estudo foi desenvolvido na Região de Saúde Estrada de Ferro Macrorregião Centro Sudestes de Goiás (RSEF-MCSG), composta pelos municípios de Anhanguera, Caldas Novas, Campo Alegre de Goiás, Catalão, Corumbaíba, Cumari, Davinópolis, Goiandira, Ipameri, Marzagão, Nova Aurora, Ouvidor, Palmelo, Pires do Rio, Rio Quente, Santa Cruz de Goiás, Três Ranchos e Urutaí. Dentre os quais a menor população é de 1.137 habitantes, em Anhanguera, e a maior população é 106.618 habitantes, em Catalão, segundo a estimativa do IBGE para 2018 (BRASIL, 2010).

Em decorrência das modificações resolutivas sobre a responsabilidade do controle vetorial da DC, algumas mudanças sobre coleta e análise de vetores foram observadas nas últimas duas décadas. Com o intuito de acompanhar o controle vetorial na região, dados referentes à captura e análise de triatomíneos no município pólo da RSEF-MCSG, foram analisados. Os primeiros dados disponíveis foram referentes ao ano de 2001 até 2010. As informações das capturas nos anos de 2011 a 2014 não foram encontrados, devido mudanças normativas e seus reflexos em longo prazo na organização e atribuição das gerências regionais. A partir de 2015 os 
dados referentes a tal controle ficaram vinculados à RSEF-MCSG. No entanto, apenas sete municípios (Anhanguera, Corumbaíba, Davinópolis, Nova Aurora, Ouvidor, Palmelo e Três Ranchos) reiniciaram as atividades relacionadas ao controle vetorial de Chagas.

O controle das doenças transmitidas por vetores a RSEF-MCSG seguiu o manual de Normas Técnicas de Vigilância da Doença de Chagas emitido pelo Sistema de Informação de Agravos de Notificações (SINAN). Os agentes de combate às endemias (ACE) estavam treinados para captura dos triatomíneos encontrados e as providências a serem tomadas quando são capturados. A vigilância entomológica era ocorrente em todo território da zona rural dos municípios pertencentes à RSEF-MCSG de forma ativa, quando o ACE fez vistorias nas Unidades Domiciliares (UD) e de forma passiva, quando as secretárias municipais de saúde foram procuradas pela população na suspeita da presença do invertebrado.

As UD's eram constituídas por dois ambientes, intradomiciliar representado pela residência dos moradores e peridomiciliar constituída de anexo (casa de máquinas, chiqueiro, depósitos, galinheiro, entre outros ambientes). No intradomicílio os ACE vistoriaram atrás de quadros, móveis encostados nas paredes, frestas em portas, embaixo de colchões e lugares que possam ser esconderijos para o inseto. Enquanto no peridomicílio a vigilância era maior, pois nas fazendas há um grande número de anexos, o ambiente perfeito para o desenvolvimento dos triatomíneos desde que tenha alimento suficiente.

Quando encontrados, os triatomíneos foram acondicionados em coletores de plástico, emitido um boletim da residência sobre a contaminação contendo o nome da localidade, a atividade que o agente de saúde estava executando na UD, quantidade de anexos, tipo de domicílio e onde foi capturado o triatomíneo.

Os triatomíneos, quando em condições viáveis, foram enviados para laboratório e testados quanto à positividade para $T$. cruzi, classificados por espécie e estágio. Logo após o término das análises os resultados foram enviados para o RSEF-MCSG.

No intuito de realizar um levantamento epidemiológico da infestação de triatomíneos na zona rural dos municípios pertencentes à RSEF-MCSG, os dados referentes ao vetor e sua localização foram fornecidos pelo Departamento de Controle de Vetores de Catalão (DECOVE) correspondente ao período de 2001 a 2010 e pelos demais município, jurisdicionados à RSEF-MCSG, que reiniciaram suas atividades de controle de vetores para DC, correspondente ao período de 2015 a 2018.

\section{RESULTADOS}

Durante o período de janeiro 2001 a dezembro 2010 a vigilância entomológica para DC no município de Catalão realizou vistorias em 289 localidades rurais e 11645 imóveis foram vistoriados, no ambiente intra e peridomiciliar. De todas as UD vistoriadas 107 estavam positivas para presença de triatomíneos. Sendo capturados 310 triatomíneos dos quais 253 foram examinados, representando $81,6 \%$ do total.

Como demonstrado na Tabela 1, dentre os triatomíneos capturados, sete espécies foram identificadas: Panstrongylus diasi, Panstrongylus geniculatus, Panstrongylus megistus, Rhondnius neclectus, Triatoma circumaculata, Triatoma pseudomaculata e Triatoma sordida. Comparando os indivíduos capturados no 
ambiente intradomiciliar e peridomiciliar observou-se maior infestação pela espécie $P$. megistus representando $85,8 \%$ dos indivíduos capturados.

TABELA 1 - Diferença de infestação peridomiciliar e intradomiciliar de triatomíneos capturados e examinados no período de 2001 a 2010, na Zona Rural do município de Catalão - GO.

\begin{tabular}{|l|c|c|c|c|c|c|c|c|c|c|c|c|c|}
\hline & \multicolumn{4}{|c|}{ Intradomiciliar } & \multicolumn{4}{c|}{ Peridomiciliar } & \multicolumn{5}{c|}{ Total } \\
\hline & capturado & examinado & \multicolumn{2}{|c|}{ capturado } & \multicolumn{2}{|c|}{ examinado } & \multicolumn{2}{c|}{ capturado } & \multicolumn{2}{c|}{ examinado } \\
\hline Espécies & $\mathrm{n}$ & $\%$ & $\mathrm{n}$ & $\%$ & $\mathrm{n}$ & $\%$ & $\mathrm{n}$ & $\%$ & $\mathrm{n}$ & $\%$ & $\mathrm{n}$ & $\%$ \\
\hline Panstrongylus diasi & 1 & 0,3 & 0 & 0,6 & 2 & 0,6 & 1 & 0,3 & 3 & 1,0 & 1 & 0,3 \\
\hline Panstrongylus geniculatus & 2 & 0,6 & 0 & 0,0 & 5 & 1,6 & 3 & 1,0 & 7 & 2,3 & 3 & 1,0 \\
\hline Panstrongylus megistus & 14 & 4,5 & 11 & 3,5 & 252 & 81,3 & 212 & 68,4 & 266 & 85,8 & 223 & 71,9 \\
\hline Rhondnius neclectus & 1 & 0,3 & 2 & 0,6 & 22 & 7,1 & 13 & 4,2 & 23 & 7,4 & 15 & 4,8 \\
\hline Triatoma circumaculata & 4 & 1,3 & 4 & 1,3 & 0 & - & 0 & 0,0 & 4 & 1,3 & 4 & 1,3 \\
\hline Triatoma pseudomaculata & 0 & - & 0 & 0,0 & 1 & 0,3 & 1 & 0,3 & 1 & 0,3 & 1 & 0,3 \\
\hline Triatoma sordida & 1 & 0,3 & 1 & 0,3 & 5 & 1,6 & 5 & 1,6 & 6 & 1,9 & 6 & 1,9 \\
\hline Total & $\mathbf{2 3}$ & $\mathbf{7 , 4}$ & $\mathbf{1 8}$ & $\mathbf{6 , 5}$ & $\mathbf{2 8 7}$ & $\mathbf{9 2 , 6}$ & $\mathbf{2 3 5}$ & $\mathbf{7 5 , 8}$ & $\mathbf{3 1 0}$ & $\mathbf{1 0 0}$ & $\mathbf{2 5 3}$ & $\mathbf{8 2 , 3}$ \\
\hline
\end{tabular}

As espécies capturadas foram classificadas quanto ao estágio evolutivo, sendo $178(57,4 \%)$ adultos e 132 (42,6\%) ninfas. Ambos os estágios foram encontrados com maior frequência no peridomicílio, $163(52,6 \%)$ adultos e 123 $(39,7 \%)$ ninfas. No intradomicílio foram encontrados 15 adultos $(4,5 \%)$ e nove ninfas de triatomíneos (2,9\%). Do número total de exemplares capturados, $286(92,6 \%)$ encontravam-se no peridomicílio e $24(7,7 \%)$ no intradomicílio.

A Tabela 2 demonstra o número de triatomíneos capturados por ano na pesquisa ativa entre os anos de 2001 a 2010 no município de Catalão classificados quanto ao local de captura, peridomicílio ou intradomicílio. Nessa análise o peridomicílio destaca-se novamente como o ambiente mais infestado pelo vetor. Em relação ao período de captura de triatomíneos, o ano de 2001 apresentou maior incidência e o ano de 2002 a menor.

TABELA 2 - Triatomíneos capturados pela pesquisa ativa no ambiente intradomiciliar e peridomiciliar durante os anos de 2001 a 2010, na Zona Rural do município de Catalão - GO.

\begin{tabular}{|c|c|c|c|c|c|c|c|c|c|c|c|c|c|c|c|c|c|c|c|c|}
\hline & \multicolumn{2}{|c|}{2001} & \multicolumn{2}{|c|}{2002} & \multicolumn{2}{|c|}{2003} & \multicolumn{2}{|c|}{2004} & \multicolumn{2}{|c|}{2005} & \multicolumn{2}{|c|}{2006} & \multicolumn{2}{|c|}{2007} & \multicolumn{2}{|c|}{2008} & \multicolumn{2}{|c|}{2009} & \multicolumn{2}{|c|}{2010} \\
\hline & $\mathrm{n}$ & $\%$ & $\mathrm{n}$ & $\%$ & $\mathrm{n}$ & $\%$ & $\mathrm{n}$ & $\%$ & $\mathrm{n}$ & $\%$ & $\mathrm{n}$ & $\%$ & $\mathrm{n}$ & $\%$ & $\mathrm{n}$ & $\%$ & $\mathrm{n}$ & $\%$ & $\mathrm{n}$ & $\%$ \\
\hline Intra & 1 & 0,5 & 0 & 0 , & 0 & 0,0 & 0 & 0,5 & 6 & 0,5 & 1 & 0,5 & 0 & 0,0 & 0 & 0,0 & 4 & 2,0 & 2 & 10 \\
\hline Peri & 55 & 27,4 & 11 & 5,5 & 33 & 16,4 & 17 & 8,5 & 23 & 11,4 & 9 & 4,5 & 32 & 15,9 & 21 & 10,4 & 41 & 20,4 & 12 & 6,0 \\
\hline Total & 56 & 27,9 & 11 & 5,5 & 33 & 16,4 & 17 & 8,5 & 29 & 14,4 & 10 & 5,0 & 32 & 15,9 & 21 & 10,4 & 45 & 22,4 & 14 & 7,0 \\
\hline
\end{tabular}

Durante o período de 2015 a 2018 o PCDCh foi realizado o controle vetorial em sete municípios de forma passiva ou ativa na RSEF-MCSG (Anhanguera, Corumbaíba, Davinópolis, Nova Aurora, Ouvidor, Palmelo e Três Ranchos). A vigilância entomológica vistoriou 370 localidades rurais e 1328 UD foram vistoriados, no ambiente intra e peridomiciliar, sendo 15 UD estavam positivas para presença de triatomíneos. Capturando 56 triatomíneos dos quais 10 foram examinados, representando $16,4 \%$ do total.

A Tabela 3 apresenta os triatomíneos capturados no ambiente peridomiciliar e intradomiciliar no período de 2015 a 2018, na Zona Rural dos municípios jurisdicionados RSEF-MCSG, classificados em quatro espécies: $P$. diasi, $P$. megistus, $R$. neclectuse $T$. sordida. Comparando os indivíduos capturados no 
ambiente intra e peridomiciliar verificou-se uma maior infestação da espécie $P$. megistus representando $50 \%$ dos indivíduos capturados.

TABELA 3 - Diferença de infestação peri e intradomiciliar de triatomíneos capturados e examinados no período de 2015 a 2018, na Zona Rural dos municípios jurisdicionados a Região de Saúde Estrada de Ferro - Macrorregião Centro Sudeste de Goiás.

\begin{tabular}{|c|c|c|c|c|c|c|c|c|c|c|c|c|}
\hline \multirow{3}{*}{ Espécies } & \multicolumn{4}{|c|}{ Intradomiciliar } & \multicolumn{4}{|c|}{ Peridomiciliar } & \multicolumn{4}{|c|}{ Total } \\
\hline & \multicolumn{2}{|c|}{ capturado } & \multicolumn{2}{|c|}{ examinado } & \multicolumn{2}{|c|}{ capturado } & \multicolumn{2}{|c|}{ examinado } & \multicolumn{2}{|c|}{ capturado } & \multicolumn{2}{|c|}{ examinado } \\
\hline & $\mathrm{n}$ & $\%$ & $\mathrm{n}$ & $\%$ & $\mathrm{n}$ & $\%$ & $\mathrm{n}$ & $\%$ & $\mathrm{n}$ & $\%$ & $\mathrm{n}$ & $\%$ \\
\hline Panstrongylus diasi & 1 & 1,8 & 1 & 0,6 & 0 & 0 & 0 & 0 & 1 & 1,8 & 1 & 0,6 \\
\hline Panstrongylus megistus & 7 & 12,5 & 0 & 0 & 21 & 37,5 & 9 & 16,1 & 28 & 50,0 & 9 & 5,0 \\
\hline Rhondnius neclectus & 0 & 0 & 0 & 0 & 1 & 1,8 & 0 & 0 & 1 & 1,8 & 0 & 0 \\
\hline Triatoma sordida & 12 & 21,4 & 0 & 0 & 14 & 25,0 & 0 & 0 & 26 & 46,4 & 0 & 0 \\
\hline Total & 20 & 35,7 & 1 & 0,6 & 36 & 64,3 & 9 & 16,1 & 56 & 100,0 & 10 & 5,6 \\
\hline
\end{tabular}

Em relação ao estágio evolutivo, durante os anos de 2015 a 2018 na RSEFMCSG, 43 (76,79\%) insetos capturados eram adultos e $13(23,21 \%)$ ninfas. Ambos os estágios evolutivos foram encontrados com maior frequência no peridomicílio $28(50 \%)$ adultos e oito $(14,29 \%)$ ninfas. No intradomicílio foram capturados 15 $(26,79 \%)$ triatomíneos adultos e cinco (8,93\%) ninfas. Do número total de exemplares capturados $64,29 \%$ encontravam-se no peridomicílio e $35,71 \%$ no intradomicílio.

A Tabela 4 apresenta os dados de captura dos 56 exemplares de triatomíneos entre os anos de 2015 a 2018 na RSEF-MCSG. O ano que mais ocorreu captura de indivíduos foi 2015, sendo os anos de 2016 e 2017 os anos que menos apresentaram incidência de triatomíneos capturados. Sendo o peridomicílio o ambiente que mais se destacou na presença de indivíduos capturados.

TABELA 4 - Triatomíneos capturados pela pesquisa ativa no ambiente intradomiciliar e peridomiciliar durante os anos de 2015 a 2018, na Zona Rural dos municípios jurisdicionados à Região de Saúde Estrada de Ferro - Macrorregião Centro Sudeste de Goiás.

\begin{tabular}{lccccccccc}
\hline & \multicolumn{2}{c}{2015} & \multicolumn{2}{c}{2016} & \multicolumn{2}{c}{2017} & \multicolumn{2}{c}{2018} \\
\cline { 2 - 10 } & $\mathrm{N}$ & $\%$ & $\mathrm{n}$ & $\%$ & $\mathrm{n}$ & $\%$ & $\mathrm{n}$ & $\%$ \\
\hline Intra & 17 & 30,4 & 0 & 0,0 & 1 & 1,8 & 2 & 3,6 \\
Peri & 29 & 51,8 & 1 & 1,8 & 0 & 0,0 & 6 & 10,7 \\
\hline Total & 46 & 82,1 & $\mathbf{1}$ & 1,8 & $\mathbf{1}$ & 1,8 & $\mathbf{8}$ & 4,0 \\
\hline
\end{tabular}

Como já ressaltado, nem todos os triatomíneos capturados estavam viáveis para análise laboratorial para pesquisa de formas evolutivas de $T$. cruzi no intestino. Mesmo assim, dois triatomíneos foram classificados como positivos para o protozoário causador da DC. Ambos pertenciam à espécie $P$. megistus. Um exemplar foi capturado no ano de 2006 na zona rural do município de Catalão e o outro no ano de 2015 na zona rural do município de Ouvidor.

\section{DISCUSSÃO}

Os resultados mostraram que das sete espécies de triatomíneos coletados nas UD, $P$. megistus é o mais importante para vigilância entomológica da doença de Chagas em toda RSEF-MCSG. Os baixos índices de infecção natural por T. cruzi em 
P. megistus, comparado com outros estudos realizados no estado de Goiás, demonstram que esta não é a espécie de destaque encontrada em domicílios, mas se encontra entre a terceira e quarta mais capturada (SILVA, et al., 1995; OLIVEIRA; SILVA, 2007).

Alguns dos triatomíneos foram encontrados colonizando intradomicílio evidenciando o risco de infecção humana. Um estudo sobre a ocorrência de triatomíneos sinantrópicos no Distrito Federal nos anos de 2002 a 2010 também registrou a colonização destes locais com as espécies $P$. megistus, $T$. pseudomaculata, T. sórdida, $P$. geniculatus, $R$. neglectus e $P$. diasi. Sendo $P$. megistus a espécie com maior taxa de infecção natural por T. cruzi, como observado no presente estudo realizado na RSEF-MCSG (DIAS et al., 2011).

As UD nas zonas rurais geralmente são construídas próximas a coleções de água disponíveis (riachos, ribeirões, açudes e córregos), onde geralmente existe a presença de matas ciliares, fato que colabora para presença do $P$. megistus no perímetro da residência. Essa espécie se caracteriza por ser nativa da Mata Atlântica e com grande dispersão pelo interior do país, ao longo de bosques e matas ciliares, sendo encontrada ainda em ambientes intradomiciliares e peridomiciliares (VILLELA, et al., 2005; VILLELA, et al., 2009). O P. megistus tem uma alta suscetibilidade de infecção ao $T$. cruzi, boa capacidade de adaptação a diversos habitats e hospedeiros, sendo ainda considerada uma das principais espécies de vetor da DC (ARAÚJO et al., 2015).

O encontro de ninfas capturadas no intradomicílio e no peridomicílio evidenciam o processo de domiciliação das espécies, como já registrado sobre $P$. megistuse e T. sórdida (VILLELA, et al., 2009). Outras espécies de triatomíneos encontradas no município de Catalão foram $P$. geniculatus, $T$. sordida e $R$. neglectus. Nenhum inseto capturado dentre estas espécies estavam positivos para o protozoário $T$. cruzi, sugerindo uma origem silvestre. Porém em todo na RSEFMCSG o $T$. sordida foi o segundo mais capturado tanto no ambiente intradomiciliar e peridomiciliar destacando sua capacidade de domiciliação no decorrer dos anos. Uma vez que essa espécie encontra-se na região central do Brasil (VILLELA, et al., 2009).

Dos 18 municípios que pertencem a RSEF-MCSG, havia informações acerca dos dados sobre triatomíneos e DC em apenas sete. A carência de dados dos outros municípios pode ser explicada por não ter havido captura de triatomíneos por parte dos funcionários dos municípios, ausência de insetos presente no domicílio nestas localidades, ou mesmo a simples falta de registro junto à RSEF-MCSG (VILLELA, et al., 2009). Estas são possibilidades passíveis de ocorrer, aliadas ainda com a preocupação com outras doenças transmitidas por vetores.

A inexistência de um sistema de informação de armazenamento de dados em todos os municípios dificulta os acessos às informações referentes ao PCDCh no sudeste goiano. A mudança de gestão administrativa dos municípios também é um dos aspectos que contribui para o não armazenamento continuo desses dados.

Outra limitação encontrada neste estudo foi à perda de informações referente à infecção natural de triatomíneos. Somente $81,6 \%$ dos insetos coletados foram examinados no município de Catalão e 5,6\% no inquérito apurado pela RSEFMCSG, pois muitos morreram ou o intestino se encontrava seco antes da realização do exame. A não realização dos diagnósticos está associada com o transporte e a logística dos exemplares capturados dos municípios para RSEF-MCSG e posteriormente ao laboratório. O estudo realizado por Dias e colaboradores no 
entorno de Brasília sobre inquérito epidemiológico da DC detectou esse mesmo obstáculo quanto a transporte e logística dos triatomíneos capturados nas UD, pois foi possível examinar apenas $40 \%$ dos exemplares (DIAS, et al., 2011).

Com a descentralização do serviço de saúde e ficando a cargo dos Estados e Municípios os inquéritos epidemiológicos, trabalhos já realizados dimensionavam os possíveis problemas com esse novo modelo de gestão. Uma vez que a gestão municipal é sujeita a diversas demandas, por diferentes necessidades elencadas pela população e pelos órgãos fiscalizadores ou reguladores, as vezes não conferindo ao controle entomológico da doença de Chagas o grau de importância necessário (DIAS et al., 2011).

O risco de transmissão vetorial de $T$. cruzi para os humanos no sudeste goiano pode ser considerado baixo devido às boas condições de vida da maioria dos habitantes da região. No entanto, a presença de espécimes de $P$. megistus infectados com $T$. cruzi nos domicílios mostra que o risco de infecção ainda existe no estado de Goiás. Assim, é essencial continuar a vigilância entomológica, intensificando-a nos municípios de maior ocorrência e o fortalecimento dos Postos de Informação de Triatomíneos (PIT) como incentivo da comunidade, com base em ações, para a participação ativa na vigilância entomológica. Desta forma, a detecção de triatomíneos sinantrópicos seria mais eficiente, reduzindo as chances de colonização desses insetos em unidades domiciliares da RSEF-MCSG.

\section{CONCLUSÃO}

A Doença de Chagas ainda representa um grande desafio público no Brasil e um problema de saúde emergente. Neste contexto, as ações de educação em saúde juntamente com a vigilância entomológica devem se centralizar na promoção da qualidade de vida da população, a fim de evitar o processo de domiciliação de triatomíneos característicos exclusivamente de ambiente silvestre como ainda de impedir que vetores já confirmados se tornem o principal disseminador da DC e assumindo como habitat exclusivamente o ambiente doméstico. Os problemas encontrados para manutenção do programa de controle de vetores na região estudada devem ser contornados e analisados com maior prioridade uma vez que foi demonstrada a importância da educação da sociedade sobre o perigo do convívio com os insetos vetores. A resolução destas lacunas é importante também para a continuidade da principal medida profilática em relação a doenças tropicais promovidas pela proximidade da população e dos programas de controle de endemias, a educação em saúde.

\section{REFERÊNCIAS}

ARAÚJO, A. C.; RODRIGUES, S. C. R.; REZENDE, A. F. S.; VILLELA M. M.; BORSUK, S. Soroprevalência de infecção humana por Trypanosoma cruzi em uma área rural do sul do Brasil. Revista de Patologia Tropical, v. 44, n. 4, 423-43, 2015. Disponível em: doi: 10.5216/rpt.v44i4.39230.

ARRUDA, A. B. L.; SOUZA, Y. N. S.; GOMES, F. V. B. A. F., BARBOSA, J. L. J.; GONDIM Y. M., ARRUDA A. A. L. Prevalence of Chagas disease among blood donation candidates. Brazilian Journal of Health Review, v. 2, n. 5, 2019. Disponível em: doi:10.34119/bjhrv2n5-076. 
BRASIL. IBGE. Censo Demográfico [Internet], 2010 [citado 2018 nov 28]. Disponível em: https://cidades.ibge.gov.br/.

BRASIL. Ministério da Saúde (MS). Secretaria de Vigilância em Saúde. Coordenação-Geral de Desenvolvimento da Epidemiologia em Serviços. Guia de vigilância em saúde. Brasília: Ministério da Saúde, 2017. Disponível em: http://portalarquivos.saude.gov.br/images/pdf/2017/outubro/06/Volume-Unico2017.pdf.

BRASIL. Ministério da Saúde. Portaria no. 1.399 de 15 de dezembro de 1999 Regulamenta a NOB SUS 01/96 no que se refere às competências da União, estados, municípios e Distrito Federal, na área de epidemiologia e controle de doenças, define a sistemática de financiamento e dá outras providências. Diário Oficial da União, Brasília (DF) 1999; 15 dez Disponível em: http://www.funasa.gov.br/site/wp-content/files_mf/Pm_1399_1999.pdf.

DIAS, J. C. P.; CLÁUDIO, L. D. G.; LIMA, M. M; ALBAJAR-VIÑAS, P.; SILVA, R. A., et al. Changes in the paradigm of clinical and therapeutic management of Chagas' disease: progress and perspectives in the pursuit of comprehensive health, Epidemiologia e Serviço de Saúde, Brasília, v. 25, p. 87-90, 2016 Disponível em: doi: 10.5123/S1679-49742016000500003.

DIAS, J. C. P.; NETO V. A.; LUNA E. J. A. Mecanismos alternativos de transmissão do Trypanosoma cruzi no Brasil e sugestões para sua prevenção para sua prevenção, Journal of the Brazilian Society of Tropical Medicine, v. 44, n. 3, p. 375-379, 2011. Disponível em: doi.org/10.1590/S0037-86822011005000032.

DIAS, J. C.; DIAS, E.; NOBREGA, G. C. Long-term follow-up of a patient since the acute phase of Chagas disease (South American trypanosomiasis): further treatment and cure of the infection, Journal of Brazilian Society of Tropical Medicine, v. 48, n.5, p. 629-632, 2015. Disponível em: doi.org/10.1590/0037-8682-0073-2015.

GUARNER, J. Chagas disease as example of a reemerging parasite. Seminars in Diagnostic Pathology, v. 36, n. 3, p. 164-169, 2019. Disponível em: doi.org/10.1053/j.semdp.2019.04.008.

KRATZ, J. M. Drug Discovery for Chagas disease: A viewpoint, Acta Tropica, n. 198, p. 105-107, 2019. Disponível em: doi.org/10.1016/j.actatropica.2019.105107.

OLIVEIRA, A. W.; SILVA, I. G. Distribuição geográfica e indicadores entomológicos de triatomíneos sinantrópicos capturados no Estado de Goiás. Revista da Sociedade Brasileira de Medicina Tropical, v. 40, n. 2, p. 204-208, 2007. Disponível em: http://www.scielo.br/pdf/rsbmt/v40n2/a11v40n2.pdf.

GASPARIM, A. Z.; FONTES, C. E. R.; ROSSONI, D. F.; TOLEDO, M. J. O. Epidemiological and clinical profile of patients with Chagas disease in the CentralNorth area of Paraná, Southern Brazil. Revista da Sociedade Brasileira de Medicina Tropical, v. 51, n. 2, p. 225-230, 2018. Disponível em: doi.org/10.1590/0037-8682-0173-2017. 
GOIÁS - Secretaria Estadual de Saúde. [Internet]. 2018 [citado 2018 nov 28] (Disponível em: http://www.saude.go.gov.br/regional/regional-estrada-de-ferro/)

SILVA, I. G.; SILVA, J. L.; CAMARGO, M. F.; ELIAS, C. N.; SANTOS, A. H.; et al. Infestação de vetores da tripanossomíase americana no ambiente domiciliar no Estado de Goiás. Revista de Patologia Tropical, v. 24, n. 1, p.41-47, 1995 Disponível em: http://200.137.217.156/bitstream/ri/11650/2/Artigo\%20\%20lonizete\%20Garcia\%20da\%20Silva\%20-\%201995.pdf.

SILVA, T. P. T. Eliminação da transmissão da doença de Chagas pelo Triatoma infestans no Brasil: um fato histórico. Revista da Sociedade Brasileira de Medicina Tropical, v.39, $\quad$ n.5, 2006. Disponível em: http://www.scielo.br/pdf/rsbmt/v39n5/a18v39n5.pdf.

VILLELA, M. M.; Souza, J. M. B.; Melo, V. P.; Dias, J. C. P. Avaliação do Programa de Controle da Doença de Chagas em relação à presença de Panstrongylus megistus na região centro-oeste do Estado de Minas Gerais, Brasil. Cadernos de Saúde Pública, v. 25, n. 4, p. 907-917, 2009 Disponível em: doi.org/10.1590/S0102$311 \times 2009000400022$.

VILLELA, M.M.; SOUZA, J. B.; MELLO, V. P.; AZEREDO, B. V. M.; DIAS, J. C. P. Vigilância entomológica da doença de Chagas na região centro-oeste de Minas Gerais, Brasil, entre os anos de 2000 e 2003. Cadernos de Saúde Pública, v. 21, n. 3, p. 878-886, 2005. Disponível em: http://www.scielo.br/pdf/csp/v21n3/22.pdf.

WHO - World Health Organization, Chagas disease (American trypanosomiasis) [Internet]. 2018 [citado 2018 nov 12]. Disponível em http://www.who.int/mediacentre/factsheets/fs340/en/index.html 